\title{
A tale of two superpotentials: Stability and instability in designer gravity
}

\author{
Aaron J. Amsel, ${ }^{1, *}$ Thomas Hertog, ${ }^{2, \dagger}$ Stefan Hollands, ${ }^{3, *}$ and Donald Marolf ${ }^{1, \S}$ \\ ${ }^{1}$ Physics Department, UCSB, Santa Barbara, California 93106, USA \\ ${ }^{2}$ Theory Division, CERN, CH-1211 Geneva 23, Switzerland \\ and APC, 10 rue Alice Domon et Léonie Duquet, 75205 Paris, France \\ ${ }^{3}$ Inst. f. Theor. Physik, Georg-August-Universität, D-37077, Göttingen, Germany
}

(Received 1 February 2007; published 5 April 2007)

\begin{abstract}
We investigate the stability of asymptotically anti-de Sitter gravity coupled to tachyonic scalar fields with mass at or slightly above the Breitenlohner-Freedman bound. The boundary conditions in these "designer gravity" theories are defined in terms of an arbitrary function $W$. Previous work had suggested that the energy in designer gravity is bounded below if (i) $W$ has a global minimum and (ii) the scalar potential admits a superpotential $P$. More recently, however, certain solutions were found (numerically) to violate the proposed energy bound. We resolve the discrepancy by observing that a given scalar potential can admit two possible branches of the corresponding superpotential, $P_{ \pm}$. When there is a $P_{-}$branch, we rigorously prove a lower bound on the energy; the $P_{+}$branch alone is not sufficient. Our numerical investigations (i) confirm this picture, (ii) confirm other critical aspects of the (complicated) proofs, and (iii) suggest that the existence of $P_{-}$may in fact be necessary (as well as sufficient) for the energy of a designer gravity theory to be bounded below.
\end{abstract}

DOI: 10.1103/PhysRevD.75.084008

PACS numbers: 04.50.+h, 04.65.+e

\section{INTRODUCTION}

It is well known that anti-de Sitter (AdS) gravity coupled to a scalar field with mass at or slightly above the Breitenlohner-Freedman bound [1] admits a large class of boundary conditions, defined by an essentially arbitrary real function $W$. For all (regular) $W$, the conserved charges are well defined and finite [2-9], despite the fact that the scalar field falls off slower ${ }^{1}$ than usual. Theories of this type have been called designer gravity theories [5], because their dynamical properties depend significantly on the choice of $W$ (see e.g. $[4,10]$ ).

In supergravity theories with a dual conformal field theory (CFT) description, the AdS/CFT duality [11,12] relates $W$ to a potential term $\int W(\mathcal{O}) d S$ in the CFT action, where $\mathcal{O}$ is the field theory operator that is dual to the bulk scalar for $W=0$ boundary conditions [13,14]. This led [5] to conjecture that (a) there is a lower bound on the gravitational energy in those designer gravity theories where $W$ is bounded from below, and that (b) the solutions locally minimizing the energy are given by the spherically symmetric, static soliton configurations found in [5].

More recently, the stability of designer gravity theories has been studied using purely gravitational arguments. In particular, a lower bound on the conserved energy in terms

\footnotetext{
*Electronic address: amsel@physics.ucsb.edu

†lectronic address: thomas.hertog@cern.ch

${ }^{\ddagger}$ Electronic address: hollands@theorie.physik.uni-goettingen. de

${ }^{\S}$ Electronic address: marolf@physics.ucsb.edu

${ }^{1}$ We define $W$ such that $W=0$ corresponds to turning off the independent subleading term in the asymptotic expansion of the scalar field. The usual boundary conditions instead keep this term and turn off the leading term.
}

of the global minimum of $W$ was rigorously proven within a specific $\mathrm{AdS}$ gravity theory by relating the Hamiltonian charges to spinor charges [6]. Arguments were given in [8] suggesting that these bounds hold more generally. However, it was subsequently discovered [15] that solutions with arbitrarily negative energy can be constructed numerically for certain theories with $W \geq 0$. This raised a puzzle, which we resolve in this paper.

Our resolution focuses on an auxiliary construct, the "superpotential" $P(\phi)$ built from the bulk scalar potential $V(\phi)$. This superpotential is an important ingredient in constructing the spinor charge. In [8], it was shown that any $V(\phi)$ satisfying the Breitenlohner-Freedman bound perturbatively admits an appropriate superpotential $P(\phi)$. It was of course recognized that the global existence of $P(\phi)$ was required for the proof of an energy bound, and that this global existence may impose constraints on $V(\phi)$.

What is interesting about the counterexamples of [15] is that they do admit a globally defined superpotential, but nevertheless violate the proposed bound. The issue turns out to be that superpotentials associated with a given $V(\phi)$ can be of two types, which we call $P_{+}$and $P_{-}$. A particular $V(\phi)$ may admit (distinct) superpotentials of both types, or it may admit only the $P_{+}$type. The proof requires existence of a $P_{-}$-type superpotential, while the examples of [15] admit only the $P_{+}$-type. ${ }^{2}$

We verify this claim below and also confirm various other details of the arguments of $[6,8]$. After briefly listing our conventions in Sec. II, we review the proof of the lower bound in Sec. II, illustrating why a $P_{-}$-type superpotential

\footnotetext{
${ }^{2}$ The fact that a given $V(\phi)$ can admit both $P_{+}$and $P_{-}$ superpotentials was used to study asymptotically AdS domain wall solutions in [16-23].
} 
is essential (and why a $P_{+}$-type superpotential is not sufficient). Section IV confirms that the counterexamples of [15] admit only $P_{+}$-type superpotentials and numerically explores the energy bound in a number of examples. We find evidence that the global existence of $P_{-}$may be necessary (as well as sufficient) for any lower bound to hold.

Section V investigates other aspects of the proof from $[6,8]$. Arguments for positivity of the spinor charge require Witten spinors, whose global existence can be difficult to demonstrate. Questions about this global existence were raised in [15]. However, we show that the argument for global existence given in [6] for a particular $P_{-}$extends to the general case. As a check, we also explicitly demonstrate the existence of Witten spinors in the context of spherical symmetry by analyzing an associated ordinary differential equation. We evaluate these spinors numerically for a particular example and use the results to check the relation between the spinor and Hamiltonian charges derived in [8]. We close with some discussion in Sec. VI.

\section{PRELIMINARIES}

Our conventions and our definition of asymptotically anti-de Sitter spacetimes follow those of $[6,8,24]$. In particular, we consider gravity theories minimally coupled to a scalar field with Lagrangian density given by

$$
\mathbf{L}=\frac{1}{2} d^{d} x \sqrt{-g}\left[R-(\nabla \phi)^{2}-2 V(\phi)\right],
$$

where we have set $8 \pi G=1$. Here the scalar potential $V(\phi)$ is of the form

$$
V(\phi)=\Lambda+\frac{1}{2} m^{2} \phi^{2}+\ldots
$$

near $\phi=0$. For simplicity we assume that $V$ is even. The constant $\Lambda$ is the cosmological constant, given by

$$
\Lambda=-\frac{(d-1)(d-2)}{2 \ell^{2}},
$$

with $\ell$ a positive length that we may set to one by rescaling the metric and scalar field. The spacetime dimension is denoted $d$, and we assume that $d \geq 4$. We will furthermore assume that the scalar field is tachyonic $\left(m^{2}<0\right)$, with mass in the Breitenlohner-Freedman range [1]

$$
m_{B F}^{2} \leq m^{2}<m_{B F}^{2}+1,
$$

where $m_{B F}^{2}=-(d-1)^{2} / 4$.

The metric of exact AdS space of unit radius (and $\phi=$ $0)$, given by

$$
d s_{0}^{2}=-\left(1+r^{2}\right) d t^{2}+\frac{d r^{2}}{1+r^{2}}+r^{2} d \omega_{d-2}^{2},
$$

is an exact solution of the theory, where $d \omega_{d-2}^{2}$ is the unitradius round metric on $S^{d-2}$. As part of our boundary conditions, we assume that the metric of a general solution asymptotically approaches (2.5) in the manner described in
$[6,8,24]$, and that the scalar field is asymptotically of the form

$$
\phi=\frac{\alpha}{r^{\lambda_{-}}}+\frac{\beta}{r^{\lambda_{+}}}+\ldots
$$

where

$$
\lambda_{ \pm}=\frac{d-1 \pm \sqrt{(d-1)^{2}+4 m^{2}}}{2} .
$$

To obtain a well-defined dynamics for the linearized theory, it is necessary to impose a boundary condition at $r=\infty$ on the scalar field, i.e. we must impose a relation between $\alpha$ and $\beta$ in (2.6). For example, one can impose $\alpha=0$, leaving $\beta$ totally unspecified. We refer to this option as "fast falloff boundary conditions." Alternatively, one may set $\beta=0$, leaving $\alpha$ unspecified. One may also impose more general boundary conditions of the form

$$
\beta \equiv \frac{d W}{d \alpha},
$$

where $W(\alpha)$ is an arbitrary smooth function. Under the AdS/CFT duality, this function $W$ appears as an additional potential term in the action for the dual field theory $[13,14]$.

\section{A TALE OF TWO SUPERPOTENTIALS}

An elegant way to prove energy bounds is Witten's spinor method [25], which proceeds by constructing a manifestly positive "spinor charge," and then comparing it to the energy of the gravitational solution. Witten's argument was originally given in the context of asymptotically flat spacetimes, but it can be generalized to the asymptotically AdS situation. When the matter satisfies the dominant energy condition apart from the negative cosmological constant term (regarded as a contribution $-\frac{\Lambda}{8 \pi G} g_{a b}$ to $T_{a b}$ ), Witten's argument may simply be modified by the addition of a term $\frac{1}{\sqrt{2(d-2)}} P \gamma_{a}$ to the covariant derivative which acts on the spinor, where $P$ is a constant proportional to $\sqrt{-} \Lambda$. This term is needed to deal with the negative energy associated with the cosmological constant.

Our interest here is in tachyonic scalars $\phi$, whose potential energy may in fact be unbounded below and does not satisfy the dominant energy condition. As shown by [26] for $d=4$ (based on [27], and extended to higher dimensions by [28]), many such settings may be addressed by generalizing the constant $P$ to a real superpotential $P(\phi)$ satisfying

$$
V(\phi)=(d-2)\left(\frac{d P}{d \phi}\right)^{2}-(d-1) P^{2},
$$

where $V(\phi)$ is the scalar potential.

Taking $\phi=0$ to be the AdS vacuum, we are interested in potentials for which $V^{\prime}(0)=0$. The value $V(0)$ then determines the cosmological constant. With fast falloff 
boundary conditions $(\alpha=0)$, the proof $[26,28]$ requires only that there be a solution to (3.1) with $P^{\prime}(0)=0$ and $P(0)>0$. Perturbatively, i.e., in the sense of formal power series, a solution of this form always exists when $V^{\prime \prime}(0)$ sets the scalar field mass to satisfy the Breitenlohner-Freedman bound. In fact, there are two such perturbative solutions:

$$
P_{ \pm}(\phi)=\sqrt{\frac{d-2}{2}}+\frac{\lambda_{ \pm}}{2 \sqrt{2(d-2)}} \phi^{2}+O\left(\phi^{4}\right),
$$

where $\lambda_{ \pm}$are given by (2.7). However, the proof of [26,28] requires that $P(\phi)$ be well defined (and real) for all $\phi$, and this imposes further restrictions on $V(\phi)$.

In both the fast $[26,28]$ and slow falloff cases $[6,8]$, the proof proceeds by using $P_{ \pm}(\phi)$ to construct a spinor charge $Q_{ \pm}$. In the fast falloff case one may show (e.g., following the basic method outlined by [29]) that $Q_{+}=E=Q_{-}$, where $E$ is the conserved energy. The proof of $[26,28]$ is identical no matter which superpotential is used, and it is sufficient that only $P_{+}$exist. ${ }^{3}$ However, in order to derive an energy bound with slower falloff conditions, $[6,8]$ assumed the existence of $P_{-}$. We verify in subsection III A below that this choice is critical in this context, and that $P_{+}$ alone does not lead to an energy bound. This turns out to resolve the issue raised in [15]. A simple example is discussed in Sec. III B.

\section{A. Choosing the right superpotential}

We now quickly repeat the derivation of the energy bound from [6,8], examining both the original argument (using $P_{-}, Q_{-}$) and an analogous argument based on $P_{+}$, $Q_{+}$. For either superpotential, the spinor charge is defined as

$$
Q=\int_{C} * \mathbf{B},
$$

where the integrand is the Hodge dual of a suitably defined Nester two-form [30]

$$
B_{c d}=\bar{\Psi} \gamma_{[c} \gamma_{d} \gamma_{e]} \hat{\nabla}^{e} \Psi+\text { H.c., }
$$

and $C=\partial \Sigma$ is a surface at spatial infinity that bounds a spacelike surface $\Sigma$. In (3.4), $\Psi$ is a Dirac spinor and the covariant derivative is defined in terms of $P\left(=P_{ \pm}\right)$as

$$
\hat{\nabla}_{a} \Psi=\nabla_{a} \Psi+\frac{1}{\sqrt{2(\bar{d}-2)}} P(\phi) \gamma_{a} \Psi .
$$

We require that the spinor field $\Psi$ approaches a covariantly constant spinor (i.e. a Killing spinor) of pure $\mathrm{AdS}, \Psi_{0}$, at

\footnotetext{
${ }^{3}$ Both $P_{+}$and $P_{-}$exist near $\phi=0$. However, a real solution ceases to exist if (3.1) forces $P^{\prime}(\phi)$ to become imaginary; i.e., if $V+(d-1) P^{2}<0$. Since $P_{+}>P_{-}$near $\phi=0$, and since this in turn implies $\left|P_{+}^{\prime}\right|>\left|P_{-}^{\prime}\right|$, one finds $\left|P_{+}\right|>\left|P_{-}\right|$for all $\phi \neq 0$ and global existence of $P_{-}$implies global existence of $P_{+}$, but not vice versa.
}

infinity. We furthermore assume that asymptotically $-\bar{\Psi} \gamma^{a} \Psi \rightarrow\left(\partial_{t}\right)^{a}$. Using Gauss's theorem we can rewrite the spinor charge $Q\left(=Q_{ \pm}\right)$as

$$
Q=\int_{\Sigma} d(* \mathbf{B})=\int_{\Sigma}\left(\nabla^{b} B_{a b}\right) u^{a} d S
$$

where $d S$ is the integration element on $\Sigma$, and $u^{a}$ the unit normal. Letting $i, j, \ldots$ denote directions in the tangent space of the surface $\Sigma$, one can then show [28] that the integrand of (3.6) is

$$
\begin{aligned}
\left(\nabla^{b} B_{a b}\right) u^{a}= & {\left[2\left(\hat{\nabla}_{i} \Psi\right)^{\dagger} \hat{\nabla}^{i} \Psi-2\left(\hat{\nabla}_{i} \Psi\right)^{\dagger} \gamma^{i} \gamma^{j} \hat{\nabla}_{j} \Psi\right.} \\
& \left.+\lambda^{\dagger} \lambda\right],
\end{aligned}
$$

where

$$
\lambda=\frac{1}{\sqrt{2}}\left(\gamma^{a} \nabla_{a} \phi-\sqrt{2(d-2)} \frac{d P}{d \phi}\right) \Psi .
$$

The first and third terms in (3.7) are manifestly nonnegative as written. A negative contribution from the second term can be avoided by imposing the Witten condition [25]

$$
\gamma^{i} \hat{\nabla}_{i} \Psi=0,
$$

which is essentially the spatial Dirac equation. In Sec. V, we recall the argument [6] that globally smooth spinors satisfying [13] with the above boundary conditions exist in designer gravity. This establishes $Q \geq 0$.

However, an energy bound can be derived only once we relate $Q$ to the physical energy $E$. While these coincide for fast falloff boundary conditions, they differ in the slow falloff case. One consequence of this is that $Q$ is not in general conserved. Another is that $Q$ may now depend on whether the spinor charge is defined using $P_{+}$or $P_{-}$. That is, $Q_{+} \neq Q_{-}$.

In [6,8], the covariant phase space method of [31-33], was used to show (following [24]) that energy in designer gravity takes the form ${ }^{4}$ :

$$
\begin{aligned}
E= & -\int_{C} \mathcal{E}_{a b} \xi^{a} d s^{b}-\left(\lambda_{+}-\lambda_{-}\right) \\
& \times \int_{C}\left[W(\alpha)-\frac{\lambda_{-}}{d-1} \alpha \beta\right] \xi_{b} d s^{b},
\end{aligned}
$$

where $\xi=\frac{\partial}{\partial t}$ is the time translation conjugate to $E, d s^{a}$ is the integration element on the cut $C \cong S^{d-2}$ of the AdS conformal boundary $I$, and $\mathcal{E}_{a b}$ is the suitably rescaled electric part of the Weyl tensor, which is smooth at $I$ as a consequence of our boundary conditions. The numerical value of $E$ is independent of the particular choice of the cut.

One can derive the relation between $Q_{ \pm}$and the energy $E[6,8]$ by expanding the metric and the spinors in an asymptotic series in $1 / r$, using the Witten equation, and

\footnotetext{
${ }^{4}$ Expression (3.10) describes the generic case. Additional terms are present for special cases. See [8]
} 
using Einstein's equation. The result is:

$$
\begin{aligned}
Q_{ \pm}= & E-\int_{C}\left[\left(\lambda_{+}-\lambda_{-}\right) W(\alpha)-\left(\lambda_{ \pm}-\lambda_{-}\right) \alpha \beta\right] d \omega \\
& +\frac{1}{2} \lim _{r \rightarrow \infty}\left(\lambda_{ \pm}-\lambda_{-}\right) r^{d-1-2 \lambda_{-}} \int_{C_{r}} \alpha^{2} d \omega,
\end{aligned}
$$

where for simplicity we have chosen $C \cong S^{d-2}$ to be a cut of constant $t$ so that $d \omega$ is the integration element of the unit sphere $S^{d-2}$. In the final term, $C_{r}$ is a large sphere of radius $r$ in $\Sigma$.

Choosing $P_{-}$and using $Q_{-} \geq 0$ yields the bound

$$
E \geq \operatorname{Vol}\left(S^{d-2}\right)\left(\lambda_{+}-\lambda_{-}\right) \inf W,
$$

where $\operatorname{Vol}\left(S^{d-2}\right)$ is the volume of the unit radius $S^{d-2}$. On the other hand, for $\alpha \neq 0$ choosing $P_{+}$causes the final term in (3.11) to diverge. Since $E$ and $W$ are manifestly finite, it follows that $Q_{+}$is infinite. It is also infinitely larger than $E$, and its positivity yields no lower bound on $E$. Thus, the energy bound (3.12) has been established only if the theory admits a $P_{-}$superpotential; the existence of $P_{+}$ alone is not sufficient.

\section{B. A simple example}

Let us consider a simple example shown in [15] to have an energy $E$ which is unbounded below. We take $d=4$ and the superpotential

$$
P(\phi)=\left(1+\frac{1}{2} \phi^{2}\right) \exp \left(-\frac{1}{16} \phi^{4}\right)
$$

This choice corresponds to $m^{2}=-2$ (that is, $\lambda_{-}=$ $1, \lambda_{+}=2$ ), and since the coefficient of $\phi^{2}$ is $\frac{1}{2}$, we see from (3.2) that (3.13) is of the $P_{+}$type. To see that the corresponding $P_{-}$does not exist, let us write (3.1) as

$$
\frac{d P}{d \phi}=\frac{1}{\sqrt{2}} \sqrt{V+3 P^{2}}
$$

One may solve (3.14) by integrating out from $\phi=0$ and matching to the expansion (3.2) for small $\phi$. Such solutions exist until the quantity $V+3 P^{2}$ becomes negative. Now, note that (3.2) implies $P_{-}<P_{+}$for all nonzero $\phi$ and similarly for their derivatives. In our example, $P_{+}$has a maximum at the global minimum of the potential occurring at $\phi=\phi_{\min }$, so $V+3 P_{+}^{2}$ vanishes there. But this means that $V+3 P_{-}^{2}$ must vanish at some $\phi_{s}<\phi_{\min }$. In fact, it must vanish linearly at $\phi_{s}$, since $P_{-}^{\prime}\left(\phi_{s}\right)=0$ would force $V^{\prime}\left(\phi_{s}\right)=0$ by (3.1). Thus a real $P_{-}$cannot exist for $\phi>$ $\phi_{s}$. This is illustrated in Fig. 1 using numerical solutions of (3.14).

Since only $P_{+}$exists for all $\phi$, one does not expect (3.12) to hold for solutions which explore large values of $\phi$. Indeed, [15] found solutions with $W \geq 0$ and arbitrarily negative energy for this potential.

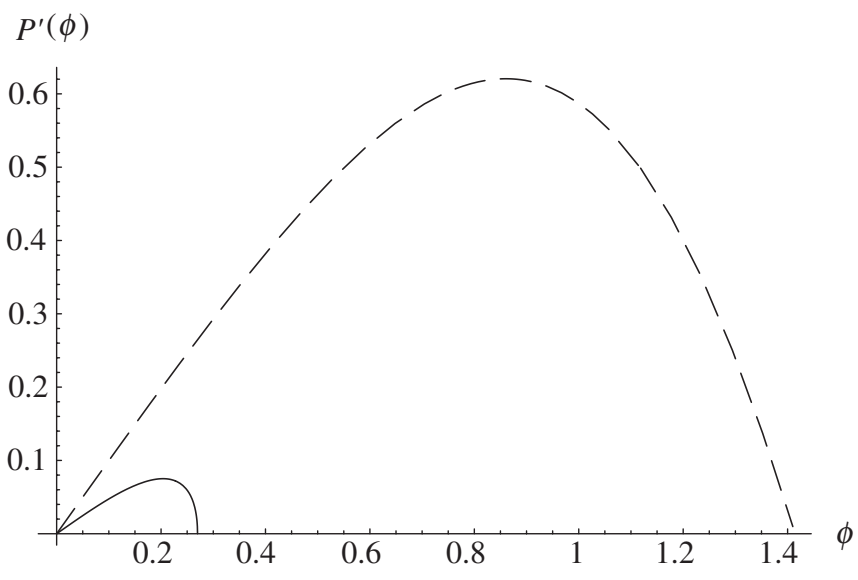

FIG. 1. Shown here are numerical plots of the derivative $P^{\prime}(\phi)$ for the example presented in the text. The dashed line corresponds to the $P_{+}$solution, which is stationary at the global minimum of the potential, $\phi_{\min }=\sqrt{2}$. The solid line corresponds to the $P_{-}$solution, whose derivative vanishes at $\phi \approx$ .27. Hence, $P_{-}$does not meet the global existence criterion.

\section{GRAVITATIONAL SOLITONS}

In order to confirm the above resolution of the puzzle raised in [15], we now numerically investigate energy bounds in a simple class of examples. For designer gravity boundary conditions that preserve the full AdS symmetry group, the existence of solitons has proven to be a reliable indicator whether or not a theory satisfies a positive energy theorem $[5,15,34,35]$. When the theory has a static spherical soliton solution, no such theorem can hold because AdS-invariant gravitational solitons can always be rescaled to obtain solutions with arbitrarily negative energy that obey the same boundary conditions. ${ }^{5}$

In this section we consider a one-parameter class of scalar potentials and construct spherical solitons numerically. We find that if the boundary conditions are specified by a $W$ which is (i) AdS-invariant and (ii) bounded below, then solitons exist if and only if $P_{-}$does not exist. This provides a strong test of the above stability proof, and it suggests that the existence of $P_{-}$is both a sufficient and a necessary condition for the energy to be bounded from below in designer gravity.

We consider the following class of potentials in $d=4$ dimensions:

$$
V(\phi)=-3-\phi^{2}-\frac{2}{3} \phi^{6}+A \phi^{8},
$$

where $A>0$ is a free parameter. These yield scalar potentials with a negative maximum at $\phi=0$, and with two global minima at $\phi= \pm \phi_{\min }$. Small fluctuations around $\phi=0$ have $m^{2}=-2$, which is within the range (2.4). Hence asymptotically the scalar generically decays as

\footnotetext{
${ }^{5} \mathrm{We}$ emphasize that the soliton itself has positive mass.
} 


$$
\phi=\frac{\alpha}{r}+\frac{\beta}{r^{2}}+\ldots
$$

and the asymptotic behavior of the $g_{r r}$ metric component reads

$$
g_{r r}=\frac{1}{r^{2}}-\frac{\left(1+\alpha^{2} / 2\right)}{r^{4}}+O\left(r^{-5}\right)
$$

To construct the corresponding superpotentials $P_{ \pm}$we solve (3.1), starting with $P(0)=1$ and with $P^{\prime \prime}(0)=\frac{1}{4} \times$ $\left(3 P(0) \pm \sqrt{9 P(0)^{2}+4 V^{\prime \prime}(0)}\right)$. A solution to (3.1) exists unless $V+3 P^{2}$ becomes negative. As we integrate out from $\phi=0, P$ is initially increasing and $V+3 P^{2}$ remains positive because the scalar satisfies the BreitenlohnerFreedman bound. For sufficiently large values of $A$ the global minima at $\pm \phi_{\min }$ will not be very much lower than the local maximum at $\phi=0$, so one expects global solutions for $P_{ \pm}$to exist, with $P_{ \pm}^{\prime}\left(\phi_{\min }\right)>0$. By contrast, if the global minima are too deep, the quantity under the square root may become negative before the global minimum is reached, and consequently a real superpotential $P$ will not exist. Clearly there are two distinct critical potentials (with parameters $A_{c}^{ \pm}$), corresponding to those where $V+3 P_{ \pm}^{2}$ just vanishes as the global minimum is reached. Numerically solving (3.1) for a range of values for $A$, one finds $A_{c}^{+}=.1$, and $A_{c}^{-}=.283$. We plot the latter critical potential in Fig. 2 (full curve). The dashed line in Fig. 2 shows a potential with $A_{c}^{+} \leq A<A_{c}^{-}$.

We now look for static spherical soliton solutions of the theory (4.1) satisfying AdS-invariant boundary conditions with $W$ bounded below. Since $\lambda_{+}=2$ and $\lambda_{-}=1$, AdSinvariant boundary conditions are given [4] by $W=k \alpha^{3}$ for real $k$. We see that $W$ is bounded below only for $k=0$,

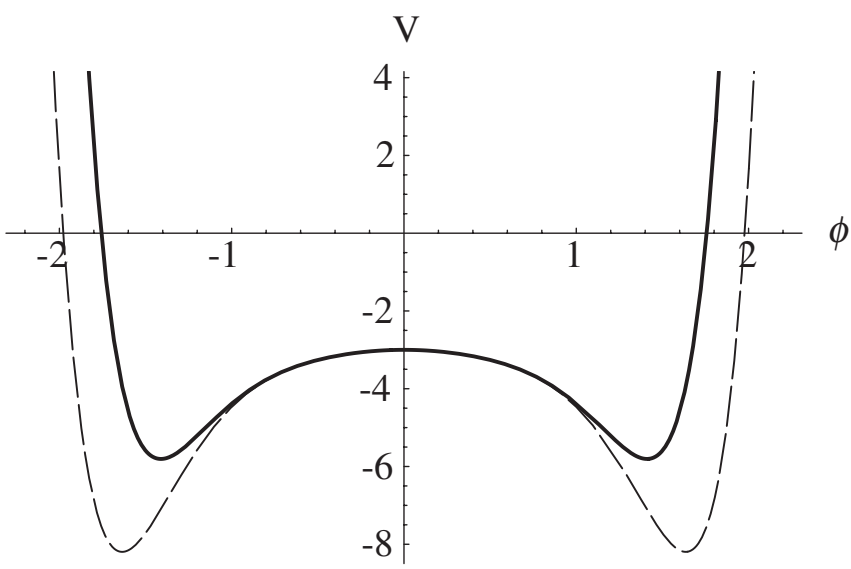

FIG. 2. The critical potential (solid line) that is on the verge of violating the positive energy theorem for $W \geq 0$ designer gravity boundary conditions. The dashed line shows a potential that violates the positive energy theorem for designer gravity boundary conditions, but satisfies such a theorem for fast falloff boundary conditions $(\alpha=0)$. so that only solitons with $\beta=0$ lead to violations of (3.12).

The metric of a general such solution takes the form

$$
d s^{2}=-h(r) e^{-2 \chi(r)} d t^{2}+h^{-1}(r) d r^{2}+r^{2} d \omega_{2}^{2},
$$

and the field equations read

$$
\begin{gathered}
h \phi_{, r r}+\left(\frac{2 h}{r}+\frac{r}{2} \phi_{, r}^{2} h+h_{, r}\right) \phi_{, r}=V_{, \phi}, \\
1-h-r h_{, r}-\frac{r^{2}}{2} \phi_{, r}^{2} h=r^{2} V(\phi), \\
\chi_{, r}=-\frac{1}{2} r \phi_{, r}^{2} .
\end{gathered}
$$

Regularity at the origin requires $h=1$ and $h_{, r}=\phi_{, r}=$ $\chi, r=0$ at $r=0$. Rescaling $t$ shifts $\chi$ by a constant, so its value at the origin is arbitrary. Thus soliton solutions can be labeled by the value of $\phi$ at the origin and the set of all soliton solutions of a particular potential with a negative maximum is found by integrating the field equations (4.5), (4.6), and (4.7) for different values of $\phi$ at the origin. For $|\phi(0)|<\phi_{\min }$ the scalar asymptotically behaves as (4.2) with constant $\alpha, \beta$. The soliton therefore defines a point in the $(\alpha, \beta)$ plane for each such $\phi(0)$. Repeating for all $\phi(0)$ yields a curve $\beta_{s}(\alpha)$. Given a choice of boundary condition $\beta(\alpha)$, the allowed solitons are simply given by the points where the soliton curve intersects the boundary condition curve: $\beta_{s}(\alpha)=\beta(\alpha)$.

Here we are interested in the existence of $\beta=0$ solitons, for potentials of the form (4.1). Figure 3 shows how the value of $\alpha$ for the $\beta=0$ soliton changes when one increases the potential parameter $A$, from $A \approx .2$ to its critical value $A_{c}^{-}$. This corresponds to deforming $V$ from the potential given by the dashed line in Fig. 2, to the critical potential at which $P_{-}$begins to exist. One sees that $\alpha \rightarrow \infty$ precisely when $A \rightarrow A_{c}^{-}$. Furthermore, for $A>A_{c}^{-}$

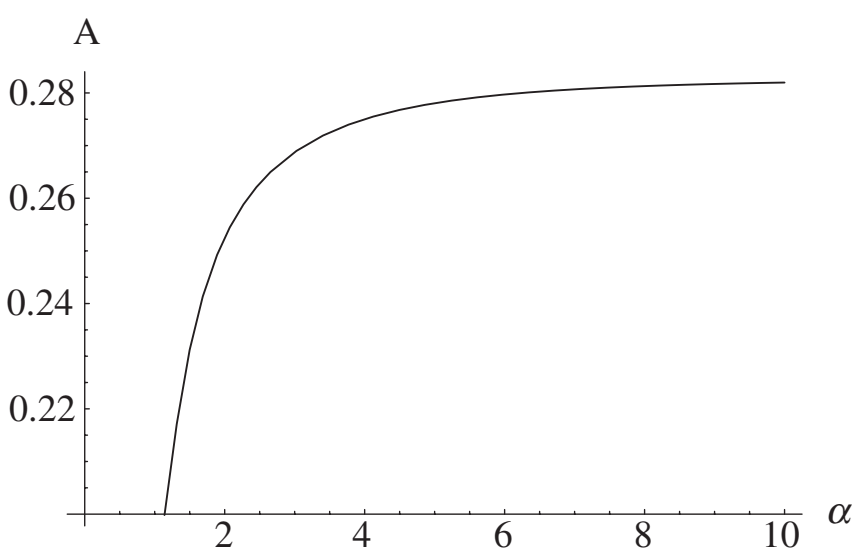

FIG. 3. The value of $\alpha$ for the $W=0$ soliton in a range of theories with different values for $A$, the coefficient of the $\phi^{8}$ term in (4.1). One sees that regular spherical $W=0$ soliton solutions cease to exist precisely when $A \rightarrow A_{c}^{-}=.283$. 
the $\beta_{s}(\alpha)$ curve intersects the $\beta=0$ axis only at the origin, so no nontrivial soliton solution exists in this parameter regime. ${ }^{6}$ It appears, therefore, that regular spherical $W=0$ soliton solutions cease to exist precisely when $A \rightarrow A_{c}^{-}$.

The existence of scalar solitons with AdS-invariant boundary conditions for $A<A_{c}^{-}$implies there are negative energy solutions in these theories. This can be seen as follows [34,36]. Starting with a static soliton $\phi_{s}(r)$, consider the one-parameter family of configurations $\phi_{\lambda}(r)=$ $\phi_{s}(\lambda r)$. Because we chose conformally invariant boundary conditions, these are again satisfied by the rescaled data. It then follows from the constraint equations that the total energy of the rescaled configurations takes the form

$$
E_{\lambda}=\lambda^{-3} E_{1}+\lambda^{-1} E_{2},
$$

where $E_{2}$ is independent of the potential and is manifestly positive, and both $E_{i}$ are finite and independent of $\lambda$. Furthermore, because the static soliton extremizes the energy [37], one has

$$
0=\left.\frac{d E_{\lambda}}{d \lambda}\right|_{\lambda=1}=-3 E_{1}-E_{2}
$$

and hence $E_{1}=-\frac{1}{3} E_{2}<0$. Therefore the contribution to the energy that scales as the volume, which includes the potential and scalar terms, is negative. This means that rescaled configurations ${ }^{7} \phi_{\lambda}(r)$ with $\lambda<1 / \sqrt{3}$ must have negative total energy, and hence violate the energy bound (3.12). Thus, the energy is unbounded below for potentials with $A<A_{c}^{-}$and $W=0$, indicating that existence of $P_{-}$is necessary for energy in designer gravity to be bounded below. (Note that we have already proven that it is sufficient.)

\section{EXISTENCE OF WITTEN SPINORS}

As explained in Sec. III, the derivation of the lower bound for the energy $E$ uses the positivity of the spinor charge. This requires the global existence of smooth spinors satisfying the Witten condition

$$
L \Psi=0
$$

on a constant time surface $\Sigma$ with suitable asymptotic behavior, where $L$ is the operator in the Witten equation,

$$
L=\gamma^{i} \hat{\nabla}_{i}=\gamma^{i}\left(D_{i}+\frac{1}{\sqrt{2(\bar{d}-2)}} P(\phi) \gamma_{i}\right)-\frac{1}{2} K \not h,
$$

\footnotetext{
${ }^{6}$ When $A$ is further decreased to $A_{c}^{+}$, an intersection point at finite $\beta$ appears between $\beta_{s}$ and the $\alpha=0$ axis. This supports the claim of [28] that the theory admits a positive energy theorem for $\alpha=0$ scalar boundary conditions only when $V$ can be derived from a superpotential $\left(P_{+}\right)$.

${ }^{7}$ The rescaled configurations $\phi_{\lambda}(r)$ are initial data for timedependent (but time-symmetric) solutions. For sufficiently small $\lambda$ one has a large central region where $\phi$ is essentially constant and away from an extremum of the potential. Hence one expects the field to evolve to a spacelike "big crunch" singularity [36].
}

$D_{i}$ is the spin-connection intrinsic to the spacelike surface $\Sigma, K$ is the trace of its extrinsic curvature, and $\not \iota=u^{a} \gamma_{a}$ is defined in terms of the unit normal to $\Sigma$.

Global existence of such solutions is nontrivial to establish, and indeed it was suggested in [15] that some subtle failure of such spinors to exist might be responsible for the lack of energy bounds in the systems studied there. In contrast, we have now proposed that the failure of those examples to satisfy (3.12) was due solely to the fact that such potentials do not admit a $P_{-}$-type superpotential. In order to dispel any remaining concerns about the global existence of spinors, we reexamine this issue below. Section VA follows [6] and proves global existence of the Witten spinors in general. This proof is however quite abstract and draws on certain very nontrivial results in the mathematics literature. We therefore restrict attention in Sec. VB to the particular case of maximal (e.g., timesymmetric), spherically symmetric hypersurfaces, which is enough to address any concerns raised by [15]. In this case we are able to give a more explicit construction of the Witten spinors by reducing the problem to ordinary differential equations. Existence of solutions is then straightforward to show. Finally, we numerically solve for the Witten spinors in Sec. VC and evaluate the corresponding spinor charges, verifying the relationship between $Q$ and $E$ derived in Sec. III.

\section{A. The general analytic proof}

Following [6], the first step in the proof is to find a formal power series solution $\eta$ to the Witten spinor equation of the form

$$
\eta \sim \Psi_{0}+r^{-1} \Psi_{1}+r^{-2} \Psi_{2}+\ldots+r^{-N} \Psi_{N}+\ldots,
$$

where $\Psi_{n} \sim r^{1 / 2}$, and where $\Psi_{0}$ is a Killing spinor in exact AdS space (i.e., $\hat{\nabla}_{a} \Psi_{0}=0$ in exact AdS with vanishing $\phi$ ) such that

$$
-\left(\frac{\partial}{\partial t}\right)^{a}=\bar{\Psi}_{0} \gamma^{a} \Psi_{0} .
$$

The spinor fields $\Psi_{1}, \Psi_{2}, \ldots$ are determined recursively by the condition that $\eta$ be a formal power series solution to the Witten equation. It can be proven that $\Psi_{n}$ are uniquely determined for $n>0$ by making a split of the Witten equation into a part containing an $r$-derivative and a part containing derivatives tangent to a sphere of constant $r$. The explicit form of the first coefficients $\Psi_{n}$ is given in $[6,8]$. The formal power series solution satisfies the equation

$$
L \eta=J,
$$

where $J$ is a smooth source vanishing faster than any inverse power of $r$ near $I$. We may terminate the expansion (5.3) at some large finite $N$, thus obtaining a $J$ vanishing 
faster than $r^{-N+1 / 2}$.

The next step is to obtain from the formal power series solution $\eta$ a global solution $\Psi$ to the Witten equation. This step requires some global analysis. The idea is to write the global solution $\Psi$ that we seek as

$$
\Psi=\eta+\mu,
$$

where $\mu$ is a smooth spinor field to be determined. Thus, $\mu$ should satisfy $L \mu=-J$, and $\mu$ should vanish at least as fast as $r^{-1 / 2}$. The global existence of such a $\mu$ can be established using the following key inequality (5.8) which holds whenever $\left(M, g_{a b}, \phi\right)$ is a solution to the Einstein equations satisfying the asymptotic conditions given in $[6,8]$. To state the inequality, consider any smooth spinor field $u$ on $\Sigma$ that vanishes outside a large sphere, and introduce the following norm on such $u$ :

$$
\|u\|^{2}=\int_{\Sigma}\left[\left(\hat{\nabla}_{i} u\right)^{\dagger} \hat{\nabla}^{i} u+\left(1+r^{2}\right)^{-1}|u|^{2}\right] d S .
$$

Then there exists a positive constant independent of $u$ such that

$$
\text { const. }^{-1}\|u\|^{2} \leq \int_{\Sigma}|L u|^{2} d S \leq \text { const }\|u\|^{2} .
$$

The proof of the inequality uses a similar technique as in the "Hardy-inequality," combined with the key identity (3.7). It may be found in [6].

We now use the inequality to establish a solution to the equation $L \mu=-J$. We will do this by first constructing a distributional solution $\mu$, and then showing that it is in fact smooth. Let $F$ be the linear functional on smooth compactly supported spinors $u$ defined by

$$
F(u)=-\int_{\Sigma} J^{\dagger} u d S
$$

Then, by the Cauchy-Schwartz inequality and the first half of the inequality (5.8), we get

$$
\begin{aligned}
|F(u)| & \leq\left(\int_{\Sigma}|u|^{2}\left(1+r^{2}\right)^{-1} d S\right)^{1 / 2}\left(\int_{\Sigma}|J|^{2}\left(1+r^{2}\right) d S\right)^{1 / 2} \\
& \leq \operatorname{const}\left(\int_{\Sigma}|L u|^{2} d S\right)^{1 / 2} .
\end{aligned}
$$

The constant is finite because by construction $J$ drops off sufficiently rapidly at infinity. We interpret this inequality as saying that $F$ is a bounded functional with respect to the positive definite scalar product given by

$$
\langle v \mid u\rangle=\int(L v)^{\dagger} L u d S .
$$

Let $H$ be the Hilbert space defined by this inner product (which, by the second half of inequality (5.8) is identical to the Hilbert space obtained from the norm $\|\cdot\|)$ ). By the Riesz representation theorem, there is hence an element $v \in H$ such that $F(u)=\langle v \mid u\rangle$ for all $u \in C_{0}^{\infty}$. Again by the inequality (5.8), every element in $H$ is locally square integrable, so, in particular, a distribution on $\Sigma$. Thus, $v$ is a solution in the distributional sense of the equation $L^{\dagger} L v=$ $-J$. Hence, $\mu=L^{\dagger} v$ is the desired distributional solution to $L \mu=-J$, and $\Psi=\eta+\mu$ is a global solution to the Witten equation.

It remains to prove that $\Psi$ is smooth, and that it satisfies the desired boundary conditions. This will follow if we can show that $\mu$ is smooth and vanishes sufficiently fast at infinity. It follows from our construction so far only that $\mu \in L^{2}\left(\Sigma,\left(1+r^{2}\right)^{-1} d S\right)$. But since $J$ is smooth and vanishes quickly, one can now use the mapping properties of the parametrix of the operator $L$ established in [38] to prove that $\mu$ is indeed smooth and vanishes sufficiently fast. The details of this argument are given in [6].

\section{B. Spherical symmetry}

We now describe the solution to the Witten equation for a slice $\Sigma$ which is both spherically symmetric and maximal (i.e., $K=0$ ) though not necessarily static. Such a slice automatically exists when the initial data are time symmetric. Since this case is sufficient to address the concerns of [15], we explain the analysis in some detail. Our conventions will be as follows. For simplicity, we restrict attention in this subsection to the case $d=4$. We denote by $\hat{a}, \hat{b}, \ldots=0,1,2,3$ indices on a flat internal space (while $a, b, \ldots=t, r, \theta, \varphi$ are spacetime indices). The gamma matrices satisfy

$$
\gamma_{\hat{a}} \gamma_{\hat{b}}+\gamma_{\hat{b}} \gamma_{\hat{a}}=2 \eta_{\hat{a} \hat{b}},
$$

and we choose the explicit matrix representation

$$
\gamma^{0}=\left(\begin{array}{cc}
0 & i I_{2} \\
i I_{2} & 0
\end{array}\right), \quad \gamma^{\hat{j}}=\left(\begin{array}{cc}
0 & i \sigma^{\hat{j}} \\
-i \sigma^{\hat{j}} & 0
\end{array}\right),
$$

where $I_{2}$ is the $2 \times 2$ identity matrix, and where $\sigma^{1}, \sigma^{2}, \sigma^{3}$ are the standard Pauli matrices.

We wish to address spherical spacetimes. Let $\Sigma$ be a maximal $(K=0)$, spherically symmetric spacelike slice on which we set $t=0$, e.g., a surface of time symmetry. Though such spacetimes are not in general static, on $\Sigma$ they may nevertheless be written in the form (4.4), which may be described by the orthonormal frame

$$
\begin{gathered}
e^{0}=\sqrt{h(r)} e^{-\chi(r)} d t, \\
e^{1}=r \sin \theta d \varphi, \\
e^{2}=r d \theta, \\
e^{3}=\frac{1}{\sqrt{h(r)}} d r .
\end{gathered}
$$

Because $K=0$, the Witten equation (5.2) only depends on the geometry intrinsic to $\Sigma$, i.e., $e^{1}, e^{2}, e^{3}$. We further assume that the scalar field on $\Sigma$ is a function of $r$ alone, and that $\phi(r), h(r)$ are known functions. The intrinsic 
covariant derivative acting on spinor fields is in general given by

$$
D_{i} \Psi=\partial_{i} \Psi+\frac{1}{4} \omega_{i}^{\hat{j} \hat{k}} \gamma_{\hat{j}} \gamma_{\hat{k}} \Psi,
$$

where the intrinsic spin connection is determined by the relation $-d e^{\hat{i}}=\omega_{\hat{k}}^{\hat{i}} \wedge e^{\hat{k}}$. For the metric (4.4), we find

$$
\begin{gathered}
\omega^{1}{ }_{2}=\cos \theta d \varphi, \\
\omega_{3}^{1}=\sqrt{h} \sin \theta d \varphi, \\
{\omega^{2}}_{3}=\sqrt{h} d \theta .
\end{gathered}
$$

With the above ingredients in hand, the Witten condition becomes the explicit equation

$$
\begin{aligned}
0= & \gamma_{3} \sqrt{h}\left(\partial_{r}+\frac{1}{r}\right) \Psi \\
& +\frac{1}{r}\left[\gamma_{2}\left(\partial_{\theta}+\frac{\cos \theta}{2 \sin \theta}\right)+\frac{1}{\sin \theta} \gamma_{1} \partial_{\varphi}\right] \Psi+\frac{3}{2} P(\phi) \Psi .
\end{aligned}
$$

Writing $\Psi$ in terms of two-component spinors as

$$
\Psi=\left(\begin{array}{l}
\Psi_{1} \\
\Psi_{2}
\end{array}\right)
$$

(5.22) implies

$$
\begin{aligned}
& 0=i\left[\sigma_{3} \sqrt{h}\left(\partial_{r}+\frac{1}{r}\right)+\frac{1}{r} \not_{S^{2}}\right] \Psi_{2}+\frac{3}{2} P(\phi) \Psi_{1}, \\
& 0=i\left[\sigma_{3} \sqrt{h}\left(\partial_{r}+\frac{1}{r}\right)+\frac{1}{r} \not_{S^{2}}\right] \Psi_{1}-\frac{3}{2} P(\phi) \Psi_{2} .
\end{aligned}
$$

Here the operator $\varnothing_{S^{2}}$ is the Dirac operator on the 2dimensional sphere $S^{2}$. To decouple the differential equations (5.23) and (5.24), we define

$$
\Psi_{+} \equiv \frac{1}{2}\left(\Psi_{1}+i \Psi_{2}\right), \quad \Psi_{-} \equiv \frac{1}{2}\left(\Psi_{1}-i \Psi_{2}\right)
$$

Then the $\Psi_{ \pm}$satisfy

$$
\begin{aligned}
& {\left[\sigma_{3}\left(\partial_{r}+\frac{1}{r}\right)+\frac{1}{r \sqrt{h}} \not_{S^{2}}+\frac{3}{2 \sqrt{h}} P(\phi)\right] \Psi_{+}=0,} \\
& {\left[\sigma_{3}\left(\partial_{r}+\frac{1}{r}\right)+\frac{1}{r \sqrt{h}} \not_{S^{2}}-\frac{3}{2 \sqrt{h}} P(\phi)\right] \Psi_{-}=0 .}
\end{aligned}
$$

Next we wish to expand $\Psi_{ \pm}$in spinor spherical harmonics, which are eigenfunctions of $\varnothing_{S^{2}}$. These are given, for example, in [39], by

$$
\begin{aligned}
\Theta_{ \pm n l}^{(-)}(\theta, \varphi)= & \left(\begin{array}{c}
\left(\cos \frac{\theta}{2}\right)^{l+1}\left(\sin \frac{\theta}{2}\right)^{l} P_{n-l}^{(l, l+1)}(\cos \theta) \\
\pm i\left(\cos \frac{\theta}{2}\right)^{l}\left(\sin \frac{\theta}{2}\right)^{l+1} P_{n-l}^{(l+1, l)}(\cos \theta)
\end{array}\right) \\
& \times e^{-i(l+(1 / 2)) \varphi},
\end{aligned}
$$

$\Theta_{ \pm n l}^{(+)}(\theta, \varphi)=\left(\begin{array}{c}i\left(\cos \frac{\theta}{2}\right)^{l}\left(\sin \frac{\theta}{2}\right)^{l+1} P_{n-l}^{(l+1, l)}(\cos \theta) \\ \pm\left(\cos \frac{\theta}{2}\right)^{l+1}\left(\sin \frac{\theta}{2}\right)^{l} P_{n-l}^{(l, l+1)}(\cos \theta)\end{array}\right) e^{i(l+(1 / 2)) \varphi}$,

where $n, l$ are integers such that $n, l \geq 0$ and $n \geq l$. The $P_{n}^{(a, b)}(x)$ are Jacobi polynomials and the spherical harmonics satisfy

$$
\not_{S^{2}} \Theta_{ \pm n l}^{(s)}= \pm i(n+1) \Theta_{ \pm n l}^{(s)} .
$$

Separating variables as

$$
\begin{aligned}
& \Psi_{+}(r, \theta, \varphi)=\sum_{s, \pm, n, l} R_{ \pm n l}^{(s)}(r) \Theta_{ \pm n l}^{(s)}(\theta, \varphi), \\
& \Psi_{-}(r, \theta, \varphi)=\sum_{s, \pm, n, l} \tilde{R}_{ \pm n l}^{(s)}(r) \Theta_{ \pm n l}^{(s)}(\theta, \varphi),
\end{aligned}
$$

we find from (5.26) and (5.27) that the radial functions satisfy the following differential equations:

$\frac{d R_{\mp n l}^{(s)}}{d r}+\frac{1}{r} R_{\mp n l}^{(s)}+\frac{3}{2 \sqrt{h}} P(\phi) R_{ \pm n l}^{(s)} \pm i(n+1) \frac{1}{r \sqrt{h}} R_{ \pm n l}^{(s)}=0$,

$\frac{d \tilde{R}_{\mp n l}^{(s)}}{d r}+\frac{1}{r} \tilde{R}_{\mp n l}^{(s)}-\frac{3}{2 \sqrt{h}} P(\phi) \tilde{R}_{ \pm n l}^{(s)} \pm i(n+1) \frac{1}{r \sqrt{h}} \tilde{R}_{ \pm n l}^{(s)}=0$.

To pick the desired solution to the Witten equation, we now impose our boundary conditions. We demand that our solution be regular in the interior, and that it asymptotically approaches a suitable Killing spinor $\Psi_{0}$ of pure AdS. As we will see shortly, this $\Psi_{0}$ has only $n=l=0$ components. Since the remaining modes are decoupled, they may be consistently set to zero. ${ }^{8}$ Thus we consider only $n=$ $l=0$ below.

Near the origin $r=0$, the solutions behave as

$$
R_{-00}^{(s)} \sim \frac{i c_{1}}{r^{2}}-i c_{2}, \quad R_{+00}^{(s)} \sim \frac{c_{1}}{r^{2}}+c_{2},
$$

where $c_{1}, c_{2}$ are constants. Regularity at the origin then requires boundary conditions of the form

$$
R_{-00}^{(s)}(0)=-i c, \quad R_{+00}^{(s)}(0)=c .
$$

The analysis for the $\tilde{R}_{ \pm 00}^{(s)}$ solutions near the origin is exactly the same. As $r \rightarrow \infty$, we require that the solution to the Witten condition approaches a Killing spinor in exact AdS space. For large $r$, solutions to (5.33) and (5.34) behave as

\footnotetext{
${ }^{8}$ In fact, a careful analysis shows that the boundary conditions force such modes to vanish.
} 
$\operatorname{Re} R_{-}(r)$

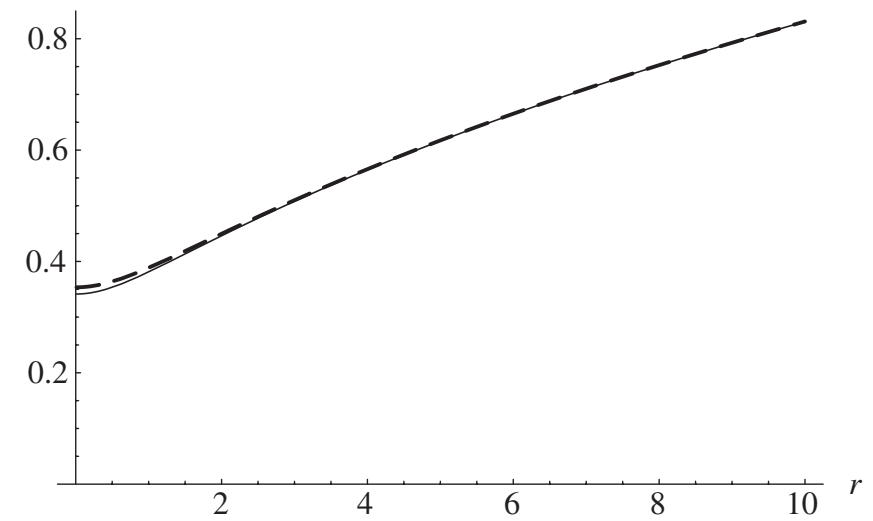

FIG. 4. Numerical results for the example (5.44) confirm the existence of Witten spinors that are regular at the origin and have the correct behavior at large $r$. The solid line is $\operatorname{Re} R_{-}=$ $\operatorname{Im} R_{+}=\operatorname{Re} \tilde{R}_{-}=\operatorname{Im} \tilde{R}_{+}$, which by the arguments in the text asymptotically approach $\frac{\rho_{1}}{2 \sqrt{2}}$, shown here as the dashed line.

$$
\begin{aligned}
R_{-00}^{(s)} \sim \frac{c_{3}}{r^{5 / 2}}-c_{4} r^{1 / 2}, & R_{+00}^{(s)} \sim \frac{c_{3}}{r^{5 / 2}}+c_{4} r^{1 / 2}, \\
\tilde{R}_{-00}^{(s)} \sim-\frac{\tilde{c}_{3}}{r^{5 / 2}}+\tilde{c}_{4} r^{1 / 2}, & \tilde{R}_{+00}^{(s)} \sim \frac{\tilde{c}_{3}}{r^{5 / 2}}+\tilde{c}_{4} r^{1 / 2} .
\end{aligned}
$$

The solution which grows as $r^{1 / 2}$ matches the known asymptotic behavior of Killing spinors in exact AdS space. In particular, an explicit solution to the Killing spinor equation is given by [40]

$$
\begin{aligned}
\Psi_{0}(t=0, r, \theta, \varphi)= & \left(\rho_{1}(r)-\rho_{2}(r) \gamma_{3}\right) \\
& \times\left(\cos \frac{\theta}{2}+\sin \frac{\theta}{2} \gamma_{3} \gamma_{2}\right) \\
& \times\left(\cos \frac{\varphi}{2}+\sin \frac{\varphi}{2} \gamma_{2} \gamma_{1}\right) U,
\end{aligned}
$$

where $U$ is a constant spinor and

$$
\begin{aligned}
& \rho_{1}(r)=\left(\frac{\left(1+r^{2}\right)^{1 / 2}+1}{2}\right)^{1 / 2}, \\
& \rho_{2}(r)=\left(\frac{\left(1+r^{2}\right)^{1 / 2}-1}{2}\right)^{1 / 2} .
\end{aligned}
$$

The spinor $U$ is chosen so that $\Psi_{0}$ satisfies the additional requirement $\left(\partial_{t}\right)^{a}=-\bar{\Psi}_{0} \gamma^{a} \Psi_{0}$, or equivalently $\Psi_{0}^{\dagger} \Psi_{0} \sim$ $r$. One can achieve this normalization by taking

$$
U=\frac{1+i}{\sqrt{2}}\left(\begin{array}{l}
1 \\
0 \\
0 \\
0
\end{array}\right)
$$

With the choice (5.40), one can rewrite (5.38) in terms of the spinor spherical harmonics as

$$
\Psi_{0}=\left(\begin{array}{c}
\frac{\rho_{1}}{\sqrt{2}}\left(\Theta_{-00}^{(-)}+i \Theta_{+00}^{(-)}\right) \\
\frac{i \rho_{2}}{\sqrt{2}}\left(i \Theta_{-00}^{(-)}+\Theta_{+00}^{(-)}\right)
\end{array}\right)
$$

Hence, the solution for the Witten spinor is

$$
\Psi=\left(\begin{array}{c}
\left(R_{-00}^{(-)}+\tilde{R}_{-00}^{(-)}\right) \Theta_{-00}^{(-)}+\left(R_{+00}^{(-)}+\tilde{R}_{+00}^{(-)}\right) \Theta_{+00}^{(-)} \\
-i\left[\left(R_{-00}^{(-)}-\tilde{R}_{-00}^{(-)}\right) \Theta_{-00}^{(-)}+\left(R_{+00}^{(-)}-\tilde{R}_{+00}^{(-)}\right) \Theta_{+00}^{(-)}\right]
\end{array}\right)
$$

Furthermore, comparing to (5.41) we see that the asymptotic conditions on the radial functions are

$$
\begin{array}{ll}
R_{-00}^{(-)} \rightarrow \frac{1}{4}(1-i) r^{1 / 2}, & R_{+00}^{(-)} \rightarrow \frac{1}{4}(i-1) r^{1 / 2} \\
\tilde{R}_{-00}^{(-)} \rightarrow \frac{1}{4}(1+i) r^{1 / 2}, & \tilde{R}_{+00}^{(-)} \rightarrow \frac{1}{4}(1+i) r^{1 / 2} .
\end{array}
$$

Comparing (5.36) and (5.43) with (5.35) and (5.37) demonstrates that the desired Witten spinors exist. One first notes that $R_{ \pm 00}^{(s)}$ decouple from $\tilde{R}_{ \pm 00}^{(s)}$. To construct the desired $R_{ \pm 00}^{(s)}$, one simply enforces the boundary condition (5.36) at $r=0$ for some $c$ and integrates (5.33) outward. From (5.37), any solution will grow asymptotically as $r^{1 / 2}$, and in fact will satisfy (5.43) up to an overall scale. Rescaling $c$ yields the desired solutions $R_{ \pm 00}^{(s)}$. The analysis for $\tilde{R}_{ \pm 00}^{(s)}$ is similar. Thus, Witten spinors exist for any spherically symmetric maximal hypersurface $\Sigma$ satisfying our boundary conditions.

\section{Numerical results}

A final crucial aspect of the argument of Sec. III is the detailed relation (3.11) between the spinor charges $Q_{ \pm}$and the energy $E$. Since (3.11) was derived via a tedious calculation, it is useful to verify the relation numerically. Let us consider the theory with potential

$$
V(\phi)=-3-\phi^{2}-\frac{2}{3} \phi^{6}+\frac{1}{2} \phi^{8},
$$

which is just (4.1) for $A=1 / 2>A_{c}^{-}$. This theory admits

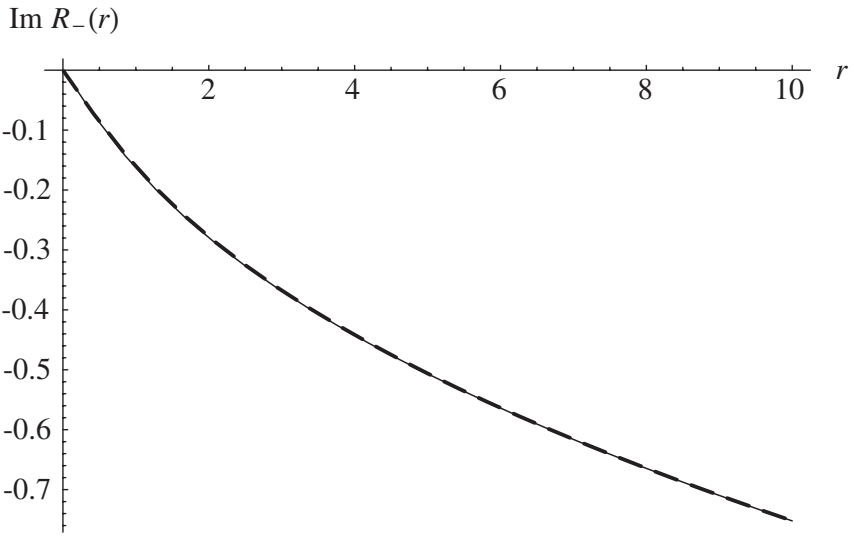

FIG. 5. The solid line is $\operatorname{Im} R_{-}=\operatorname{Re} R_{+}=-\operatorname{Im} \tilde{R}_{-}=$ $-\operatorname{Re} \tilde{R}_{+}$, which by the arguments in the text asymptotically approach $-\frac{\rho_{2}}{2 \sqrt{2}}$, shown here as the dashed line. 
both a $P_{+}$and a $P_{-}$superpotential. We take as initial data a soliton with $\phi(0)=1 / 2$ and boundary condition $W(\alpha)=$ $k \alpha$ for some constant $k \neq 0$ (so that $W$ is not conformally invariant). We find that a soliton exists with $\beta_{s}=k \approx$ -.566 and $\alpha_{s} \approx .787$. The energy of this soliton is $E \approx$ -2.956 .

Numerical results for $P_{-}$-type Witten spinors are shown in Figs. 4 and 5. ${ }^{9}$ Using these solutions, we can also calculate the spinor charge and check (3.11). We obtain $Q_{-} \approx 2.655$, thus confirming the relation $E=$ $Q_{-}+4 \pi W$. Similarly, for the $P_{+}$case we find $\left(Q_{+}-E+\right.$ $4 \pi(W-\alpha \beta)) /\left(2 \pi \alpha^{2} r\right) \approx 1.00$, in agreement with (3.11).

\section{DISCUSSION}

We have resolved the puzzle raised in [15] concerning energy bounds in designer gravity. While the arguments of $[6,8]$ are correct as written, global existence of an appropriate superpotential is a subtle requirement. In particular, two types of superpotentials may (or may not) exist for a given scalar potential $V(\phi)$. The proof of $[6,8]$ requires the global existence of a $P_{-}$-type superpotential. If one attempts to follow the same argument with a $P_{+}$-type superpotential, one finds that the difference between the conserved energy $E$ and the associated spinor charge $Q_{+}$ diverges, and, in particular, that $Q_{+}$diverges. Thus, positivity of $Q_{+}$does not yield a lower bound for $E$, and the existence of a $P_{+}$-type superpotential alone is not sufficient to yield a positive energy theorem. Numerical explorations support this resolution.

A specific question raised in [15] concerned the global existence of Witten spinors. We have demonstrated (Sec. V) that no such difficulties arise, even for the models considered in [15]. The existence theorem of [6] was shown to hold in general and, for the special case of spherical symmetry, existence of Witten spinors on maxi-

\footnotetext{
${ }^{9}$ In theories containing AdS-invariant solitons, we have also verified numerically the existence of the Witten spinors (for $P_{+}$) with initial data given by the rescaled configurations $\phi_{\lambda}(r)=$ $\phi(\lambda r)$ discussed in Sec. IV.
}

mal (e.g., time-symmetric) hypersurfaces was again demonstrated using simple arguments based on ordinary differential equations. The spherical time-symmetric context is sufficient to address the concerns of [15]. This reinforces our claim that, when $W$ has a global minimum, the existence of a $P_{-}$-type superpotential is sufficient for the energy to be bounded below.

Interestingly, our numerical explorations of particular examples (Sec. IV) suggest that global existence of $P_{-}$ may also be necessary for the energy to be bounded below. Such a property would be analogous to the claim of [28] that the existence of a superpotential is necessary for an energy bound to hold in the case of fast falloff boundary conditions $(\alpha=0)$. However, in the fast falloff case the relevant superpotential is $P_{+}$, since either superpotential is sufficient and existence of $P_{-}$implies existence of $P_{+}$ (footnote 3). Indeed, for $\alpha=0$ the spinor charges $Q_{ \pm}$ satisfy $Q_{ \pm}=E$ and, in particular, $Q_{+}=Q_{-}$when both potentials exist. Thus we find that each type of superpotential provides a stability criterion for AdS gravity-scalar theories, with $P_{+}$controlling the $\alpha=0$ case and $P_{-}$ controlling cases with slower falloff conditions on the scalar.

Finally, a key tool in our investigation of particular examples was the argument from [5,15,34,35] showing that the existence of designer gravity solitons satisfying AdS-invariant boundary conditions implies that the energy is unbounded below. We may thus interpret the lower bounds of $[6,8]$ in terms of the soliton content of such theories. We conclude that designer gravity theories where $P_{-}$exists can have no solitons when $W$ is both AdSinvariant and bounded below.

\section{ACKNOWLEDGMENTS}

The authors would like to thank Gary Horowitz for useful discussions. S.H. would also like to thank Bob Wald for discussions and the physics department at UCSB for its hospitality. A. A. and D. M. were supported in part by NSF Grant No. PHY0354978, and by funds from the University of California.
[1] P. Breitenlohner and D.Z. Freedman, Ann. Phys. (N.Y.) 144, 249 (1982); Phys. Lett. B 115, 197 (1982).

[2] M. Henneaux, C. Martinez, R. Troncoso, and J. Zanelli, Phys. Rev. D 65, 104007 (2002).

[3] M. Henneaux, C. Martinez, R. Troncoso, and J. Zanelli, Phys. Rev. D 70, 044034 (2004).

[4] T. Hertog and K. Maeda, J. High Energy Phys. 07 (2004) 051.

[5] T. Hertog and G. T. Horowitz, Phys. Rev. Lett. 94, 221301 (2005).
[6] T. Hertog and S. Hollands, Classical Quantum Gravity 22, 5323 (2005).

[7] M. Henneaux, C. Martinez, R. Troncoso, and J. Zanelli, hep-th/0603185.

[8] A. J. Amsel and D. Marolf, Phys. Rev. D 74, 064006 (2006).

[9] S. Hollands and D. Marolf, gr-qc/0611044.

[10] T. Hertog and K. Maeda, Phys. Rev. D 71, 024001 (2005).

[11] J. M. Maldacena, Adv. Theor. Math. Phys. 2, 231 (1998). 
[12] O. Aharony, S. S. Gubser, J. M. Maldacena, H. Ooguri, and Y. Oz, Phys. Rep. 323, 183 (2000).

[13] E. Witten, hep-th/0112258.

[14] M. Berkooz, A. Sever, and A. Shomer, J. High Energy Phys. 05 (2002) 034.

[15] T. Hertog, Classical Quantum Gravity 24, 141 (2007).

[16] M. Berg and H. Samtleben, J. High Energy Phys. 12 (2002) 070.

[17] D.Z. Freedman, C. Nunez, M. Schnabl, and K. Skenderis, Phys. Rev. D 69, 104027 (2004).

[18] A. Celi, A. Ceresole, G. Dall'Agata, A. Van Proeyen, and M. Zagermann, Phys. Rev. D 71, 045009 (2005).

[19] M. Zagermann, Phys. Rev. D 71, 125007 (2005).

[20] K. Skenderis and P. K. Townsend, Phys. Rev. Lett. 96, 191301 (2006).

[21] I. Papadimitriou, hep-th/0606038.

[22] K. Skenderis and P.K. Townsend, Phys. Rev. D 74, 125008 (2006).

[23] K. Skenderis and P. K. Townsend, hep-th/0610253.

[24] S. Hollands, A. Ishibashi, and D. Marolf, Classical Quantum Gravity 22, 2881 (2005).

[25] E. Witten, Commun. Math. Phys. 80, 381 (1981).

[26] W. Boucher, Nucl. Phys. B242, 282 (1984).
[27] G. W. Gibbons, C. M. Hull, and N. P. Warner, Nucl. Phys. B218, 173 (1983).

[28] P. K. Townsend, Phys. Lett. B 148, 55 (1984).

[29] S. Davis, Phys. Lett. B 166, 127 (1986).

[30] J. Nester Phys. Lett. A 83, 241 (1981).

[31] J. Lee and R. M. Wald, J. Math. Phys. (N.Y.) 31, 725 (1990).

[32] V. Iyer and R. M. Wald, Phys. Rev. D 50, 846 (1994).

[33] R. M. Wald and A. Zoupas, Phys. Rev. D 61, 084027 (2000).

[34] M. Heusler and N. Straumann, Classical Quantum Gravity 9, 2177 (1992); M. Heusler, J. Math. Phys. (N.Y.) 33, 3497 (1992).

[35] T. Hertog, Phys. Rev. D 74, 084008 (2006).

[36] T. Hertog and G. T. Horowitz, J. High Energy Phys. 07 (2004) 073.

[37] D. Sudarsky and R. M. Wald, Phys. Rev. D 46, 1453 (1992).

[38] R. Mazzeo, Commun. Partial Differ. Equ. 16, 1615 (10) (1991); J. Diff. Geom. 28, 309 (1988).

[39] R. Camporesi and A. Higuchi, J. Geom. Phys. 20, 1 (1996).

[40] M. Henneaux and C. Teitelboim, Commun. Math. Phys. 98, 391 (1985). 